\title{
ESTUDIO EMPÍRICO SOBRE LA INCIDENCIA DEL LÉXICO EN LA COMPRENSIÓN DE LA LENGUA EXTRANJERA
}

\author{
EMPIRICAL STUDY ON THE ACQUISITION OF VOCABULARY IN
} A FOREIGN LANGUAGE

\section{RAÚL RUIZ CECILIA}

Universidad de Granada. Granada, España.

raulruiz@ugr.es

\section{RESUMEN}

Este artículo expone la importancia que el léxico desempeña en la comprensión e interpretación de un texto en lengua extranjera (inglés). Para ello, un grupo de estudiantes de la Universidad de Granada se sometió a un programa de lectura organizado por el grupo de investigación HUM-457 Didáctica de la Lengua y la Literatura. Al finalizar el estudio, los participantes dejaron patente que sin un mínimo de cobertura léxica (96\%) es muy difícil acceder al nivel comprensivo y/o interpretativo. Además, se recogen las estrategias que se ven obligados a utilizar para sortear las barreras léxicas y aproximarse conceptualmente al texto.

Palabras clave: Lectura, léxico, interpretación, comprensión, lengua extranjera.

\section{ABSTRACT}

This paper examines the importance of vocabulary in the comprehension and interpretation of L2 texts for the language learner. In order to achieve this, a group of students from the University of Granada (Spain) was asked to participate in a reading program organized by the Research Group HUM-457 Language and Literature Teaching. Consequently, the participants highlight that a minimum lexical coverage (96\%) is needed if attempts are made to access the text from a comprehensive and/or interpretative perspective. In addition, we collect the reading strategies they are forced to use for avoiding lexical barriers.

Keywords: Reading, lexis, interpretation, comprehension, foreign language.

Recibido: 20/04/2011. Aceptado: 18/03/2013. 


\section{INTRODUCCIÓN Y OBJETIVOS}

E estudio del léxico se erige dentro de la enseñanza de lenguas extranjeras como un pilar básico en la consecución y consolidación de la competencia comunicativa. Sin palabras, el proceso comunicativo-oral se ve severamente modificado $\mathrm{y}$ dependiente del lenguaje no verbal (y de la habilidad expresiva del emisor). Por otro lado, si se centra el proceso comunicativo en las destrezas escritas (expresión y comprensión escrita), el vocabulario recobra su función dominante y nos (in) habilita para acceder al estadio interpretativo.

$\mathrm{Al}$ enfrentarse a un texto en lengua extranjera, el sujeto tendrá una serie de necesidades básicas. En principio, se verá obligado a esquivar y sortear demandas lingüísticas, culturales y de contenido conceptual. Enfrentarse a la lectura en lengua extranjera exige del sujeto lector una consolidada habilidad para poder acceder al contenido conceptual del texto. Laufer y Sim (1985a y 1985b) determinaron que, de entre todas las características de los textos, el vocabulario se presentaba como el principal escollo a superar por los lectores en LE. Seguidamente, establecieron el contenido conceptual como el segundo problema más importante para comprender el texto y las estructuras sintácticas como el tercer factor a tener en cuenta. Junto a este papel demandador de la lectura, otra vertiente de la misma actúa como proveedora de conocimiento para superar todas estas barreras. Es decir, la lectura presenta una serie de demandas al lector y, a su vez, las suple con interesantes ofertas que repercutirán en la construcción de un sujeto lector.

De esta forma, el vocabulario, en palabras de Day y Bamford (2000: 17) es "the most difficult aspect of reading for language learners [...]. Students need help both in understanding unknown words and in building their vocabularies". El especialista en lengua extranjera tiene que ser consciente del papel que desempeña el vocabulario en la lectura y hacer todo lo posible por incrementarlo. El vocabulario será la guía imprescindible en la aventura de cualquier lector que decida enfrentarse a textos en otra lengua diferente a la materna. Las palabras nos habilitan para leer y nos cargan de una libertad sin precedentes para desenvolvernos cómodamente entre las líneas del texto. El problema se agudiza cuando los aprendices de lenguas extranjeras no tienen el vocabulario suficiente para llegar a la comprensión y posterior interpretación del texto. Necesitamos palabras para leer, pero si no leemos difícilmente incrementaremos nuestro vocabulario pasivo tan imprescindible a la hora de desentramar los entresijos y encantos que esconden las lecturas.

Los objetivos de este artículo se estructuran en torno a la importancia del léxico en el aprendizaje de lenguas enmarcados en un programa de lectura del grupo de investigación HUM-457 financiado por la Junta de Andalucía (España). Este grupo se centra en todos los aspectos didácticos y metodológicos relacionados con la enseñanza de lenguas extranjeras y materna. Dentro del mismo, una de las líneas de trabajo está dirigida hacia los procesos lectores que el aprendiz de lenguas 
activa para el acceso al significado textual. Sería en esta área donde los resultados que aquí se presentan cobran sentido y se justifican. Así, se propone realizar un estudio empírico para comprobar cómo la dificultad léxica afecta la comprensión e interpretación del texto.

\section{MARCO CONCEPTUAL}

A la hora de estudiar el vocabulario y su repercusión en la comprensión de un texto, se debe partir de la idea de que el léxico está íntimamente ligado con la cultura en la cual se ha desarrollado y enriquecido con matices semánticos (algunas veces intraducibles). Docente y discente tienen que ser conscientes de las connotaciones léxico-culturales inherentes para que la transferencia a lengua materna sea lo más aproximada posible.

Una vez señalada la relación tan estrecha existente entre léxico y herencia cultural, se reseña los principales estudios que se han centrado en destacar la importancia que el léxico desempeña en la adquisición, comprensión e interpretación de una lengua extranjera.

Desde un punto de vista cognitivo, parece que no existen diferencias acuciantes entre un sujeto lector avanzado en lengua extranjera y un sujeto lector avanzado en lengua materna. La necesidad de un vocabulario rico y amplio es imperativa en ambos sujetos. Por otro lado, los lectores iniciales de lengua extranjera se encuentran en clara desventaja con respecto a sus homólogos en lengua materna. Les falta, esencialmente, la enorme cantidad de vocablos que el hablante ha adquirido en su infancia y que activan al aprender a leer en la lengua materna (Gil Flores, 2011, ha concluido recientemente una publicación donde se pone de manifiesto la relación entre los hábitos lectores y el desarrollo de la L1). El lector de lengua extranjera parte de cero, su vocabulario se construye, altera y complementa diariamente. Poco a poco, este proceso constructivo nutrirá al estudiante-lector de suficiente conocimiento como para superar las principales barreras léxicas que impone el texto y le dotará de herramientas necesarias para teñir el proceso de lectura de autenticidad en términos de acceso a la información escrita (Golberg, Paradis y Crago, 2008). Autores como Ulijn y Kempen (1976) ya señalaron a finales de los 70 que esta falta de vocabulario es la única diferencia palpable entre el sujeto lector nativo y el no nativo. Las lagunas conceptuales no siempre son elementos diferenciales entre ambos sujetos ya que éstas dependen del conocimiento del mundo y de la experiencia que cada uno de ellos tenga.

Pero, ¿cuál es el nivel de dificultad al que debe enfrentarse el sujeto lector? Dependiendo del objetivo que se persiga, el nivel se situará un poco por encima o un poco por debajo del conocimiento real del alumno. De todos los objetivos que se pueden perseguir cuando se lee un texto en lengua extranjera, nosotros nos 
centraremos en dos: desarrollar la fluidez lectora e incrementar el léxico receptivo y productivo del lector.

Para conseguir el primer objetivo, según Samuels (1994) y Webb (2008), el estudiante-lector tiene que ser expuesto a grandes cantidades de input escrito que se encuentren un poco por debajo de su nivel real de dominio de la segunda lengua. De esta forma, el sujeto lector tendrá la posibilidad de consolidar el vocabulario ya aprendido y podrá concentrarse en el desarrollo de la fluidez. Esta zona en la cual los lectores se desenvuelven cómodamente sin necesidad de conflictos cognitivos, se conoce como comfort zone (Day y Bamford, 2000: 91). Los estudiantes leen en armonía con su competencia lingüística y lectora lo cual contribuye a aumentar la confianza en sí mismos y la motivación intrínseca. Ellos se sienten capaces de realizar prácticas lectoras en otro idioma con resultados similares a los que obtienen cuando lo hacen en su primera lengua. Con este tipo de lectura se cumple el fin último y más importante de la lectura extensiva, involucrar a los estudiantes en la historia para que disfruten con lo que leen y sean capaces de compartir e intercambiar el mayor número posible de experiencias con sus compañeros. Si se produjese el efecto contrario, la lectura carecería de sentido pues despertaría sensaciones de aburrimiento y desinterés que desembocarían en el fracaso. Nation (2001) expone que:

For fluency development, learners need to read texts that contain little or no unknown vocabulary. Unknown vocabulary slows down learners' reading and makes it more difficult to gain the smoothness and flow needed for pleasurable reading. If graded readers are used, learners should be reading very easy texts, at least one level below their present vocabulary knowledge (150).

Este planteamiento, que bien podría resumirse con la fórmula $i$ minus 1 contrasta con la hipótesis del input comprensivo propuesta por Krashen (1982) para la adquisición/aprendizaje de lenguas extranjeras. Krashen abogaba por dar a los estudiantes material lingüístico que se encontrara un poco por encima de su nivel de tal forma que se produjera progreso en el aprendizaje (input +1 ). Esta hipótesis está más en consonancia con el objetivo segundo de la lectura referido anteriormente. La filosofía que subyace al planteamiento defendido principalmente por Samuels (1994) es desarrollar automaticidad en la lectura (es decir, convertir a los estudiantes en lectores fluidos). La exposición reiterada a vocablos familiares hace que se refuerce su aprendizaje y se reconozcan rápidamente en futuras apariciones. Además, la repetición de cada palabra en contextos diferentes enriquece los matices semánticos del término y hace más precisa la expresión oral y escrita del estudiante. Day y Bamford (2000: 17) estiman que este tipo de lecturas a $i$ minus 1 pueden llegar a incrementar el vocabulario general del estudiante.

El otro objetivo que se desprende de los programas de lectura en LE es el de 
incrementar el vocabulario (pasivo o activo) del sujeto lector. El hecho de que leer contribuye a la mejora de otras destrezas lingüísticas ha sido repetido centenares de veces y subyace en cualquier programa de animación a la lectura para jóvenes. En la lengua materna, nuestro dominio léxico se enriquece y complementa a través de las numerosas exposiciones al material impreso. El contexto se convierte en nuestro más fiel aliado para deducir el significado de aquellos términos que se cruzan por primera vez en nuestro camino. El lector de LE, para poder hacer uso de esta estrategia, necesita de un control léxico lo suficientemente amplio como para valerse de la información que le proporciona el contexto. Una vez que el sujeto lector desarrolle cómodamente la competencia lectora en LE, posiblemente sea ahí cuando esté preparado para enfrentarse a textos $i$ plus 1.

Carver argues that for learners to use reading to increase their vocabulary size they need to read material that is not too easy for them, otherwise they will meet few unknown words. Easy reading may increase depth of vocabulary knowledge, but it is unlikely to increase breadth of vocabulary knowledge. Learners need to know a substantial amount of vocabulary in order to read unsimplified material, especially academic text (Nation, 2001: 148).

Por otro lado, debemos preguntarnos qué nivel de dificultad debe tener el texto y cuándo éste deja de ser "legible" y se convierte en "incomprensible". Tal vez, la respuesta más acertada sea cuando interfiera con la comprensión e interpretación del texto. En las últimas décadas, ha habido un creciente interés en determinar el umbral de dominio léxico necesario para poder acercarse a la comprensión del contenido del texto. Una vez se logra alcanzar ese nivel, el sujeto lector se convierte en independiente y es capaz de hacer progresos considerables siempre y cuando esté expuesto a cantidades óptimas de lecturas en LE.

Estudiosos de esta cuestión (Nation, 2001; Waring y Takaki, 2003; Nation y Coady, 1988; Carver, 1994; Pérez Basanta 2005; Grabe y Stoller, 2001; Laufer y Ravenhorst-Kalovski, 2010) consideran que el lector debe conocer alrededor del $96 \%$ de los vocablos que aparecen en el texto para evitar interferencias con el procesamiento del texto.

$[\ldots]$ it is well known that until the learner reads at a very high level of text comprehension and text coverage little new vocabulary can be guessed from context [...] The optimal rate seems to be between 96 to $99 \%$ coverage of known words. Secondly, learners need to meet an unknown word many times before it is learned (Waring y Takaki, 2003: 135).

En términos cuantificables, este $96 \%$ se traduce en una palabra desconocida por cada veinte conocidas (o un vocabulario de 5.000 palabras según Cobb y 
Horst, 2004). Un estudio más reciente llevado a cabo por Nation (2006) sostiene que se necesitan entre 8.000 y 9.000 vocablos para que la interpretación del texto en la lengua extranjera sea óptima. En una investigación realizada por $\mathrm{Hu}$ and Nation (2000) se concluyó que con un 96\% de dominio léxico, sólo algunos estudiantes lograron una buena comprensión. Con un $90 \%$ (una palabra desconocida por cada diez conocidas) de conocimiento del vocabulario, un mínimo porcentaje comprendió el texto y, finalmente, con un $80 \%$ (una palabra desconocida cada cinco conocidas) ningún estudiante consiguió interpretar el texto. Así, según $\mathrm{Hu}$ y Nation, el umbral de dominio léxico que aleja al lector en lengua extranjera de una mínima comprensión se sitúa en el $80 \%$ de familiaridad léxica.

Tabla I. Dominio léxico.

\begin{tabular}{|c|c|c|c|}
\hline $\begin{array}{c}\text { Cobertura } \\
(\text { coverage }) \\
\%\end{array}$ & $\begin{array}{c}\text { en No de } \\
\text { palabras } \\
\text { desconocidas }\end{array}$ & Comprensión & Interpretación \\
\hline 96 & $1 / 20$ & Buena & Normal \\
\hline 90 & $1 / 10$ & Regular & Mínima \\
\hline 80 & $1 / 5$ & Mínima & Mala \\
\hline
\end{tabular}

Carver (1994: 432) utiliza el porcentaje de palabras desconocidas en un texto para establecer los niveles de dificultad. Así, estima que el material de lectura considerado fácil se debe acercar lo más posible al $0 \%$ de desconocimiento léxico. Más de $2 \%$ de desconocimiento léxico es la línea divisoria para textos con una dificultad considerable. La ratio (entre vocabulario conocido y desconocido) se tiene que situar en torno al $1 \%$.

Tabla II. Niveles de dificultad.

\begin{tabular}{|l|c|}
\hline \multicolumn{1}{|c|}{ DIFICULTAD } & \% de desconocimiento léxico \\
\hline Fácil & $0 \%$ \\
\hline Media & $1 \%$ \\
\hline Difícil & $2 \%$ \\
\hline
\end{tabular}

En cualquier texto encontramos palabras frecuentes (aquellas que se encuentran entre los 2.000-3.000 vocablos más usuales) y palabras no frecuentes. Freebody y Anderson (1983) realizaron una investigación para sacar conclusiones en 
torno al efecto que ejercen las palabras poco frecuentes en la comprensión de la lectura. Para tal empresa colocaron vocabulario poco usual en partes importantes del texto. Aunque es complicado generalizar, parece ser que la comprensión decaía de manera considerable.

Otra área de interés se centraría en cuánto vocabulario se aprende leyendo. La respuesta a esta cuestión es muy amplia y está condicionada por múltiples factores ambientales y contextuales. No obstante, en un estudio llevado a cabo por Waring y Takaki (2003: 148), se demostró que los lectores aprendían una nueva palabra por cada hora de lectura. Hay que tener en cuenta que el sujeto lector deduce por el contexto muchas de las palabras que aparecen en la lectura pero esa deducción tiene tan sólo un carácter hipotético y funcional. Es decir, se aventura el significado con la finalidad de poder continuar en el proceso de lectura, pero sólo una mínima parte de ese vocabulario entrará a formar parte del léxico del sujeto lector. Esos términos, en futuras apariciones, posiblemente desencadenen un sentimiento de familiaridad que propicie un potencial aprendizaje.

Con respecto a la cantidad de lectura que deben realizar los sujetos, Nation (1997) señala dos factores:

a) La frecuencia con que se manifiesta el vocabulario pasivo y/o activo del lector.

b) La cantidad de tiempo que la memoria recuerda la retención de una palabra.

Siguiendo con el planteamiento de Nation, si un sujeto lector tiene un vocabulario de unas 1000 palabras y quiere pasar al siguiente nivel (1.001-2.000), las palabras tendrían que aparecer una vez cada 10.000-15.000 palabras. Si el tiempo de retención es de una semana, el aprendiz necesitará leer al menos 10.000 palabras por semana para asegurar que se produce otro encuentro con la misma palabra y así ir incrementando el vocabulario.

Otra de las cuestiones planteadas por Waring y Takaki (2003: 148) es si las palabras con mayor frecuencia de aparición en el texto tienen más posibilidades de ser aprendidas y/o adquiridas. El resultado parece evidente: cuanto más se repita la palabra, más probabilidad de entrar a formar parte del léxico pasivo del lector. No obstante, hay veces en las que la extrañeza de un término despierta la curiosidad del lector y se integra más fácilmente en su campo léxico.

La tercera cuestión indaga sobre el ritmo al que olvidamos el vocabulario aprendido. $\mathrm{Al}$ respecto, concluyeron que alrededor de la mitad de las palabras aprendidas en la lectura se olvidan después de tres meses de no practicarlas. No obstante, casi con toda seguridad, estas palabras olvidadas volverán a entrar fácilmente en las estructuras léxicas del sujeto lector y en futuros encuentros textuales. La razón de este bajo índice de retención podría deberse a que el sujeto lector no tuvo las oportunidades necesarias para interiorizarlo e integrarlo en las estructuras cognitivas pertinentes. No obstante, según se deduce de los estudios conducidos por Waring 
y Misako, las palabras que aparecían más de quince veces tan sólo contaban con el $40 \%$ de posibilidades de ser aprendidas. Así, se necesitaría entre veinte y treinta apariciones para que el sujeto lector ampliase su volumen global de vocabulario con ese término léxico. La última hipótesis que se plantean es si diferentes tests de vocabulario presentan diferentes resultados. Después de pasar varios tipos de tests, concluyeron en la relatividad que juegan los métodos de investigación en los resultados de un estudio (Terrazas Gallego y Agustín Llach, 2009, también exponen los diferentes resultados que se extraen de los tests léxicos).

Schmidt (1990) sugiere en sus investigaciones que si se resalta la forma de la palabra se facilita la asimilación de la misma. De comprobarse que hay un relación directamente proporcional entre el destacar la forma y la adquisición, las editoriales podrían valerse de esta valiosa información en la elaboración de material escrito para LE. Así, por ejemplo, las lecturas graduadas resaltarían aquellas palabras que se consideran oportunas y necesarias en un determinado nivel de lectura. Teóricamente, las estrategias cognitivas del sujeto lector se activan ante la relevancia del término y conducen a una interiorización del significado del mismo o crean en el sujeto lector la necesidad de replantearse su aprendizaje.

Cualquier lector intermedio, en el momento de enfrentarse a un texto, tiene almacenado en sus estructuras léxico-cognitivas una cantidad considerable de vocabulario receptivo (entendido éste como aquel que no presenta obstáculos para el desarrollo normal de la actividad lectora pero que muy rara vez aparece en el discurso oral del sujeto). La mayoría de las palabras que aprendemos (y/o adquirimos) pasan antes por ser receptivas y posteriormente, una vez se crean contextos comunicativos lo suficientemente significativos, pasan al plano de lo productivo.

Lo importante de todo este debate es que, en el momento de motivar al alumnado para la lectura en LE, el docente tenga en cuenta su vocabulario real y el sight vocabulary, es decir ese vocabulario que permite reconocer las palabras automáticamente mientras se lee. Si se quiere hacer especial hincapié en determinados conceptos, una buena opción es familiarizar previamente a los sujetos lectores con aquellos que se consideren relevantes por un motivo u otro.

La deducción de palabras por el contexto es otra de las estrategias sugeridas por muchos investigadores para el aprendizaje de vocabulario. Algunas veces, el contexto es una herramienta precisa, eficaz y rápida para deducir el significado de un determinado término. En estos casos, el entorno en el que aparece el término desconocido se presenta lo suficientemente explícito y clarificador como para guiar al lector hacia la correcta decodificación léxica. En este preciso momento, el vocablo pasa a formar parte del vocabulario receptivo o pasivo del sujeto. Será a través de futuras y potenciales exposiciones al término que se emprenderá el camino hacia la producción léxica activa. 


\section{METODOLOGÍA}

\subsection{Contexto}

Este trabajo de investigación se ha realizado en la Universidad de Granada (Facultad de Ciencias de la Educación) durante el curso académico 2007-2008. Tal y como se ha expuesto en el marco teórico, la investigación que se presenta en este artículo se enmarca dentro del grupo de investigación HUM-457 Didáctica de la Lengua y la Literatura financiado por la Junta de Andalucía (España).

\subsection{Participantes}

La muestra está compuesta por ocho estudiantes de Magisterio Lengua Extranjera. Todos ellos se encontraban en el tercer y último año de sus estudios universitarios y participaron de forma voluntaria en la experiencia.

Edad: El rango de edad oscila entre los 20 y los 25 años.

Género: En la investigación participaron cinco mujeres y tres hombres reflejando, en la medida de lo posible, la realidad existente en los estudios relacionados con la educación en España (63\% mujeres y 37\% hombres).

Lengua: Los participantes comparten el español como lengua materna y su nivel de lengua extranjera oscila entre el B1 y B2 (nivel intermedio) atendiendo a los parámetros que establece el Common European Framework for Languages: Learning, Teaching, Assessment publicado por el Consejo de Europa en 2001. Para conocer la competencia lingüística en inglés de nuestra muestra, se utilizó el Oxford Placement Test (test 1).

\subsection{Procedimiento y materiales}

Este estudio se desarrolló durante los meses de diciembre a mayo del curso académico referido anteriormente. Los participantes fueron convocados a una primera reunión en la que se les explicaron detalladamente los objetivos de la investigación y cuáles serían sus obligaciones durante los meses que duraría el proyecto. Tras confirmar que estaban de acuerdo y que se comprometían a llegar hasta el final, les facilitamos el programa de lectura con las obras seleccionadas. Éstas son: $M r$. Loveday's Little Outing, de Evelyn Waugh; The Statuette, de Kenneth Ireland; y Her Father's Attic, de Philippa Pearce (2002). Los colaboradores tendrían que leer cada 
una de las lecturas en un plazo máximo de dos meses (se decidió darles un amplio margen para que cada uno accediese a los textos en el momento que considerase más apropiado y cuando ellos creyesen que podrían disfrutarlos más). Durante el proceso lector tenían que prestar especial atención a las palabras desconocidas (señalándolas de un color diferente) y ser conscientes de las estrategias utilizadas para superar las potenciales barreras léxicas. Los textos impresos subrayados se entregaron al investigador en cada una de las reuniones-control bimensuales que se mantuvieron durante el periodo del estudio. Este material es una parte importante de la investigación ya que permitió calcular con exactitud la tasa de desconocimiento léxico que se detalla en las líneas que siguen (el investigador sabía el número total de palabras del texto al tenerlo en formato word. Este total se puso en relación aritmética con el número de palabras desconocidas que habían señalado cada sujeto en los textos).

Ahora bien, ¿cómo calcular el número de palabras conocidas y desconocidas en un texto? A continuación se propone dos fórmulas utilizadas en este estudio: La primera está pensada para calcular el número de palabras desconocidas en el texto. La segunda está orientada a obtener el porcentaje para poder compararlos con los descritos anteriormente.

1) Palabras conocidas:

número total de palabras en el texto menos palabras desconocidas

2) Porcentaje:

número de palabras conocidas en el texto X 100 el número total de palabras en el texto

Una vez aplicadas las operaciones anteriores, se procedió a clasificar los resultados según los estudios previos que aparecen en el marco conceptual de este trabajo y que se vinculan directamente con la comprensión e interpretación de textos en una lengua extranjera (la tabla con las frecuencias absolutas y relativas aparece en el Anexo I).

Asimismo, al finalizar las distintas lecturas, rellenaban un diario de lectura (reading journal) donde reflejaban su interacción con el texto y las conexiones con su experiencia previa (estos resultados serán objeto de futuras publicaciones más centradas en la parte cualitativa de la investigación). Desde un punto de vista formal, el diario de lectura propuesto se compone de cuatro estadios fundamentales: fase contextual, fase pretextual, fase textual y fase postextual. Todos ellos fueron ideados sin perder de vista al lector como auténtico protagonista del proceso de lectura. Los participantes tenían total libertad para direccionar sus reflexiones de 
la forma más significativa posible. El diario de lectura, en su formato completo, fue publicado en la revista Lenguaje y Textos, 23-24 (Ruiz-Cecilia, 2006: 187-196).

Además, también reflexionaron sobre las estrategias de lectura utilizadas y cuyas conclusiones se recogen en el siguiente apartado de este artículo (la información ha sido extraída de los diarios de lectura). Una vez los participantes entregaron todos los diarios de lectura, se mantuvieron entrevistas individualizadas con ellos para recoger feedback e intercambiar impresiones suscitadas a raíz de su implicación en la lectura de los textos. Las entrevistas se hicieron con preguntas abiertas y fueron grabadas (con permiso de todos ellos) para su posterior análisis y sobre todo para interpretar algunas de las reflexiones plasmadas en los diarios de lectura. La información sirvió para triangular los datos cuantitativos y para recabar información que pudiera ser útil en futuras publicaciones.

\section{RESULTADOS Y DISCUSIÓN}

Los resultados que se expondrán y comentarán a continuación forman parte de una investigación más amplia dirigida al desarrollo de la habilidad lectora en la lengua extranjera. Los datos que recogemos se limitan a la parte léxica de los textos.

Para analizar la dificultad léxica de las tres historias cortas se ha tenido en cuenta varias variables. Nos centramos, primeramente, en la tasa de palabras desconocidas y su relación con la comprensión de la historia expuesta en el marco conceptual de este trabajo. Por otro lado, se recoge el porcentaje de palabras desconocidas y el porcentaje de cobertura léxica (palabras conocidas) de cada uno de los sujetos. Se menciona el nivel de dificultad atendiendo a criterios descritos por Carver (1994). En otro nivel, se abordan las estrategias de resolución léxica que los sujetos lectores mencionan en sus respectivos diarios de lectura.

Para establecer conclusiones acerca de la idoneidad o no de las historias cortas que han configurado nuestro corpus, seguiremos los principios consensuados en varios de los estudios llevados a cabo (Nation y Coady, 1988; Grabe y Stoller, 2001; Carver, 1994; Nation, 2001; Waring y Takaki, 2003; Pérez Basanta, 2005; Nation, 2006).

Tal y como se comenta en el marco conceptual del presente trabajo, la comprensión de un texto en lengua extranjera se ve afectada cuando la tasa de desconocimiento es inferior a $1 / 20$, es decir, una palabra desconocida en cada veinte. Teniendo en cuenta este dato, la comprensión de nuestros ocho sujetos no debería haberse visto afectada. De todos ellos, el único que se aproxima vertiginosamente al límite es el sujeto $2(1 / 26 ; 1 / 21 ; 1 / 21)$. Tal vez por ello su diario de lectura refleje una cierta pobreza en la matización de las ideas y en la interpretación de las historias. El resto de participantes supera ampliamente el mínimo impuesto, 
encontrando algunos que llegan a $1 / 132$ (sujetos 7 y 8 en $M r$. Loveday's Little Outing). Si se toma como referente la media de cada una de las historias, se puede apreciar cómo en todas las historias hay cuatro sujetos coincidentes que se sitúan por encima de la media (sujetos 4, 5, 7 y 8 ) y otros cuatro, también coincidentes, que se encuentran por debajo de la media (sujetos 1, 2, 3 y 6). Aunque todos ellos manifiesten un nivel aceptable de conocimiento léxico en la LE para proceder a la lectura de cada una de las historias cortas, sí que se puede afirmar que los sujetos 4, 5, 7 y 8 tienen una mayor competencia en la LE que se refleja en las reflexiones plasmadas en los diarios de lectura. La media indica que el texto más fácil (léxicamente hablando) ha sido Mr. Loveday's Little Outing (1/59) y el más complicado The Statuette (1/46).

Con respecto a la cobertura léxica, se aprecia cómo no existe un paralelismo entre la tasa de palabras desconocidas y un mismo porcentaje que se repita en varias historias. Esta aparente asimetría se debe a que los datos están calculados sobre el total de palabras de cada una de las historias. Por lo tanto, el 98,9\% que el sujeto 7 tiene en Her Father's Attic (es decir 1/87) difiere con el 98,9\% que el mismo sujeto manifiesta en The Statuette (es decir 1/94). Los cálculos se deben realizar con las peculiaridades léxicas de cada una de las historias. No obstante, los diversos porcentajes (indistintamente si las historias tienen más o menos palabras) sí son comparables con el $96 \%$ consensuado por los diferentes investigadores del área.

Los datos han sido reexpresados en un gráfico de columnas apiladas donde se indica la cobertura y el porcentaje de desconocimiento. La última columna (guía) marca los porcentajes estándares.

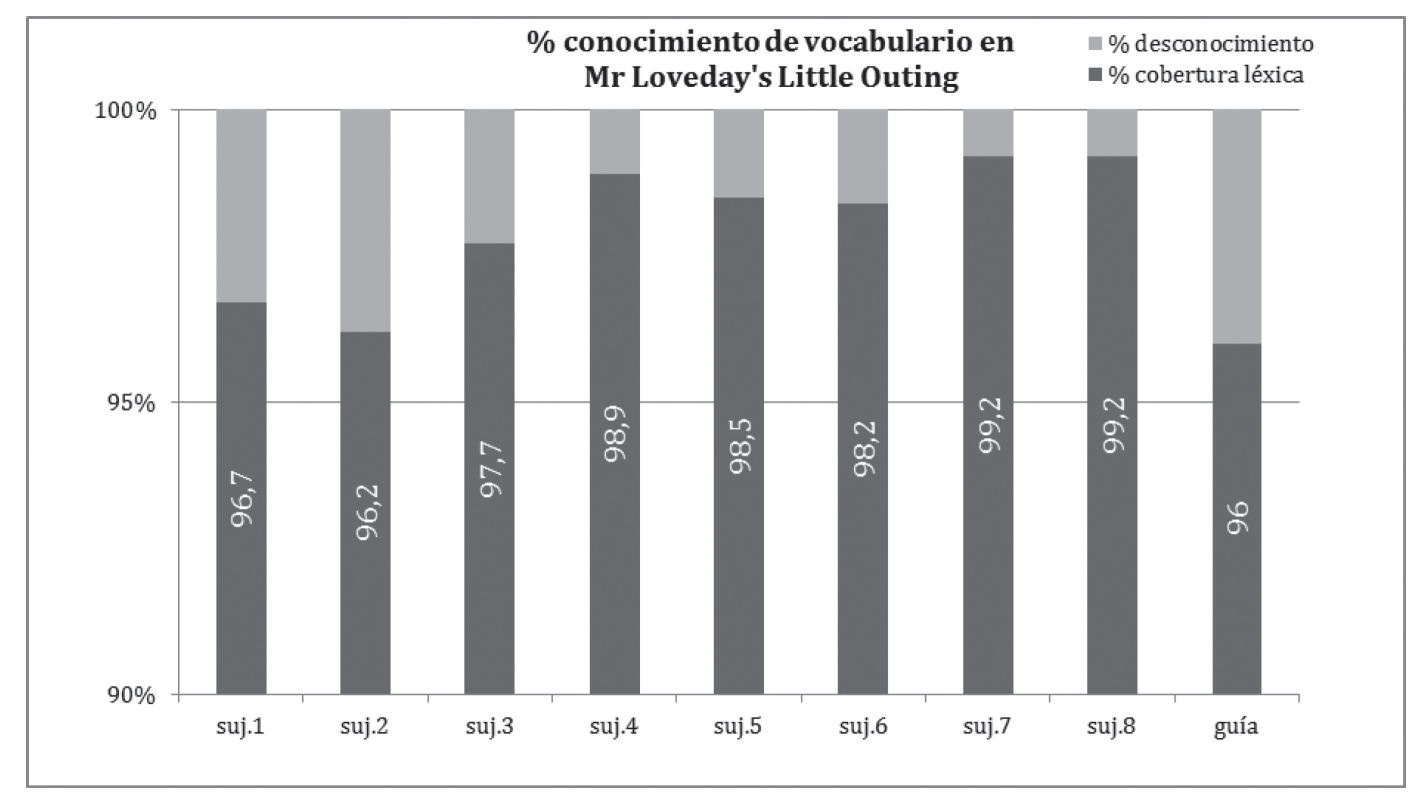

Gráfico 1. Porcentaje conocimiento de vocabulario en Mr. Loveday's Little Outing. 


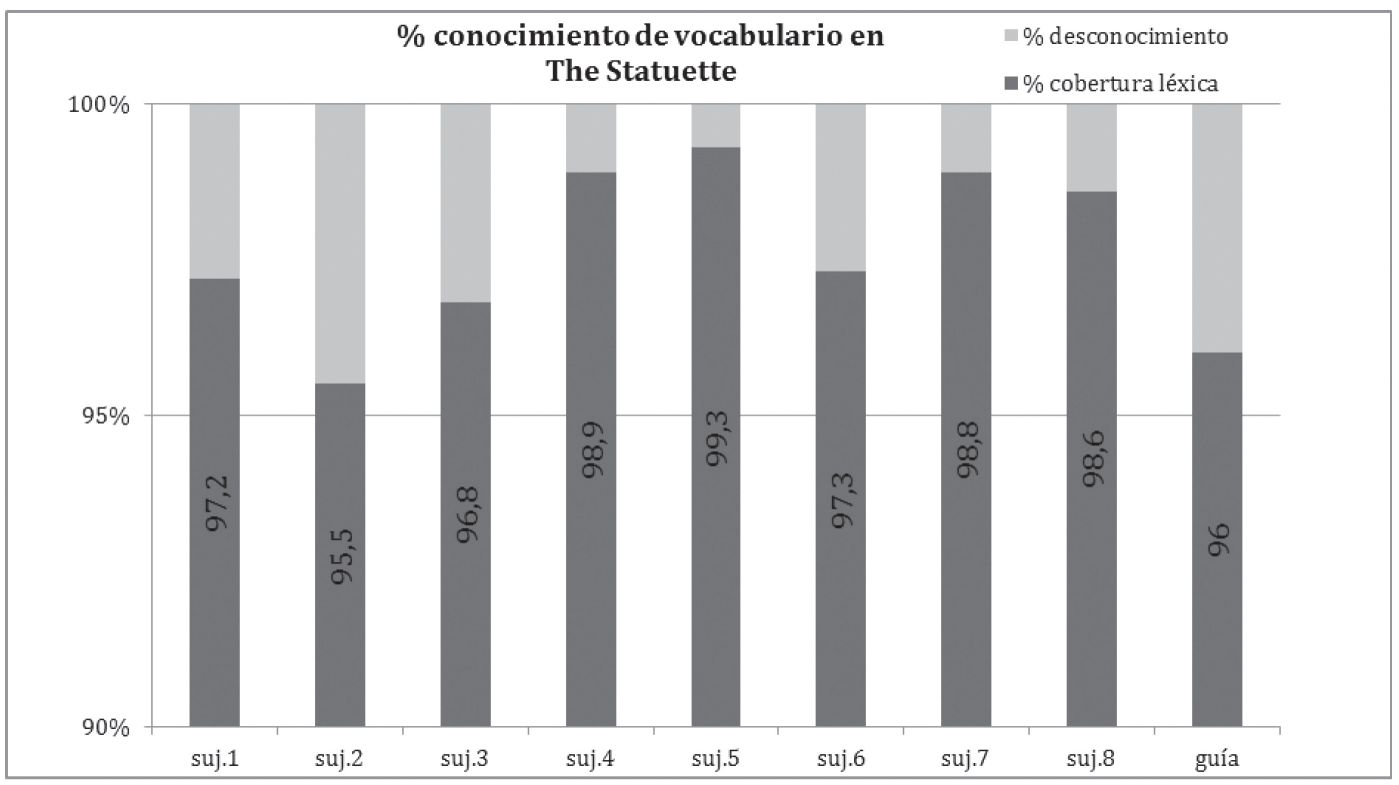

Gráfico 2. Porcentaje conocimiento en The Statuette.

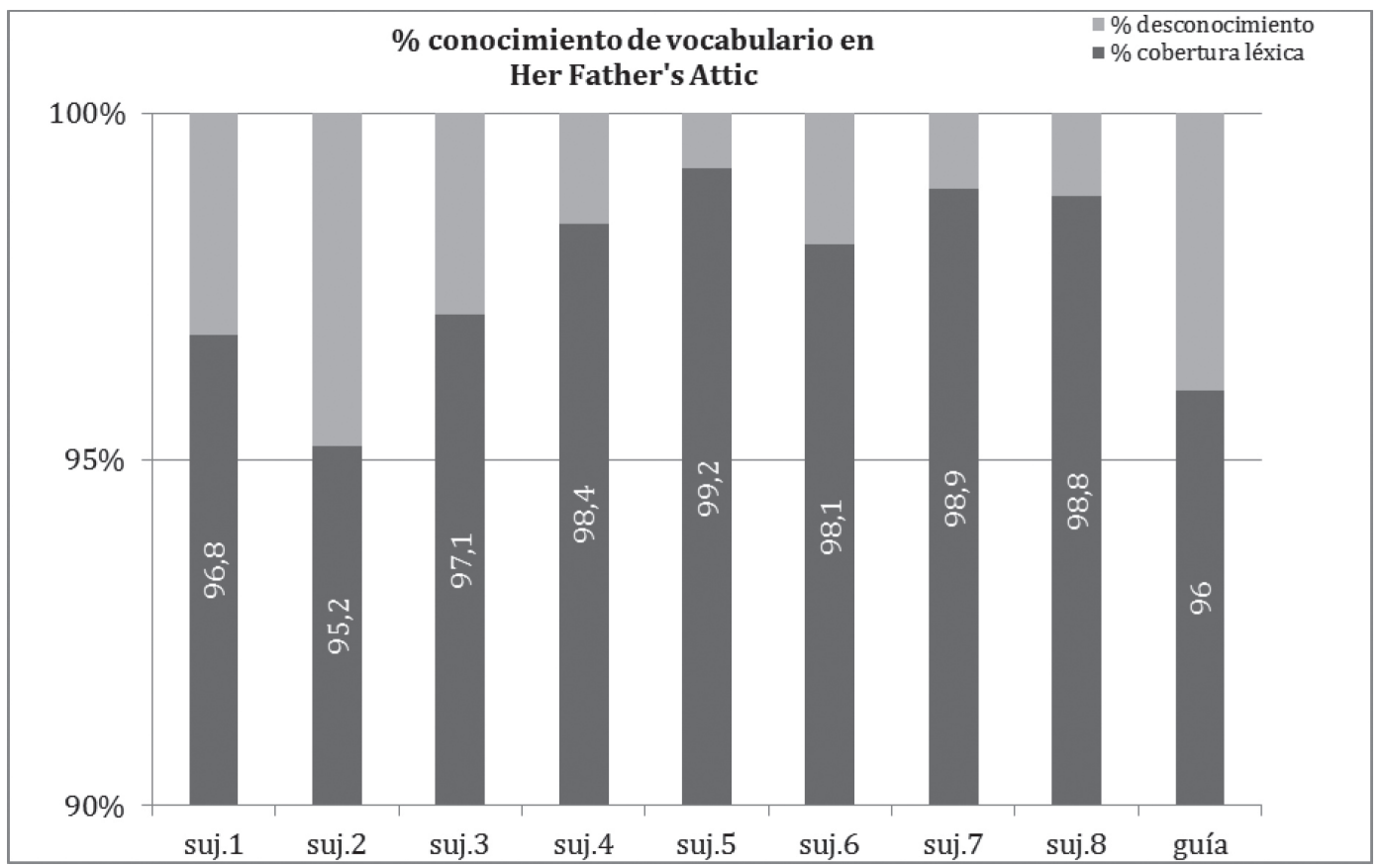

Gráfico 3. Porcentaje conocimiento en Her Father's Attic.

En las columnas apiladas de las gráficas anteriores se puede apreciar cómo la mayoría de los sujetos se sitúan por encima del $96 \%$ de cobertura léxica. La única excepción la representa el segundo sujeto que en The Statuette y en Her Father's Attic alcanza el 95,5 y el 95,2\% respectivamente. Tal y como vimos anteriormente, la comprensión se ve seriamente afectada cuando la cobertura se sitúa en torno 
al $80 \%(=1 / 5)$. El sujeto en cuestión puede acceder al contenido conceptual del texto (incluso reconoce en su diario de lectura no tener problemas de comprensión de ideas), pero con un mayor esfuerzo (materializado en el número de palabras por minuto y en el tiempo dedicado a la lectura).

\subsection{Nivel del texto según el porcentaje de desconocimiento}

Para expresar la relación entre estas dos variables, se optó por un gráfico de barras. En éste, por un lado, se encuentra el porcentaje de desconocimiento que presenta cada sujeto y el nivel de dificultad del texto, ateniéndonos al planteamiento de Carver (1994: 432) con ligeras modificaciones. Los resultados que se obtengan sólo aportan información relativa al nivel del texto sin que sea necesario un conflicto con la comprensión del mismo (comentada en la gráfica anterior).

Según Carver, un texto con un porcentaje de desconocimiento superior al 2\% es un material de lectura difícil; alrededor del $1 \%$ es un material apropiado; y con un porcentaje de $0 \%$ es una lectura fácil. Estos porcentajes se reestructuraron en cuatro categorías. Una lectura fácil es aquella cuyo porcentaje de desconocimiento se sitúa por debajo del $0,9 \%$. El material apropiado se encuentra entre un 1 y un $1,5 \%$. Tomando como referencia una de las hipótesis de Krashen (1993) para el aprendizaje de lenguas extranjeras, se consideró material de lectura un poco por encima del nivel de los estudiantes $(\mathrm{L}+1)$, aquel que alcanza desde un 1,6 hasta un $1,9 \%$ de desconocimiento. Finalmente, un texto es difícil cuando el porcentaje supera el $2 \%$.

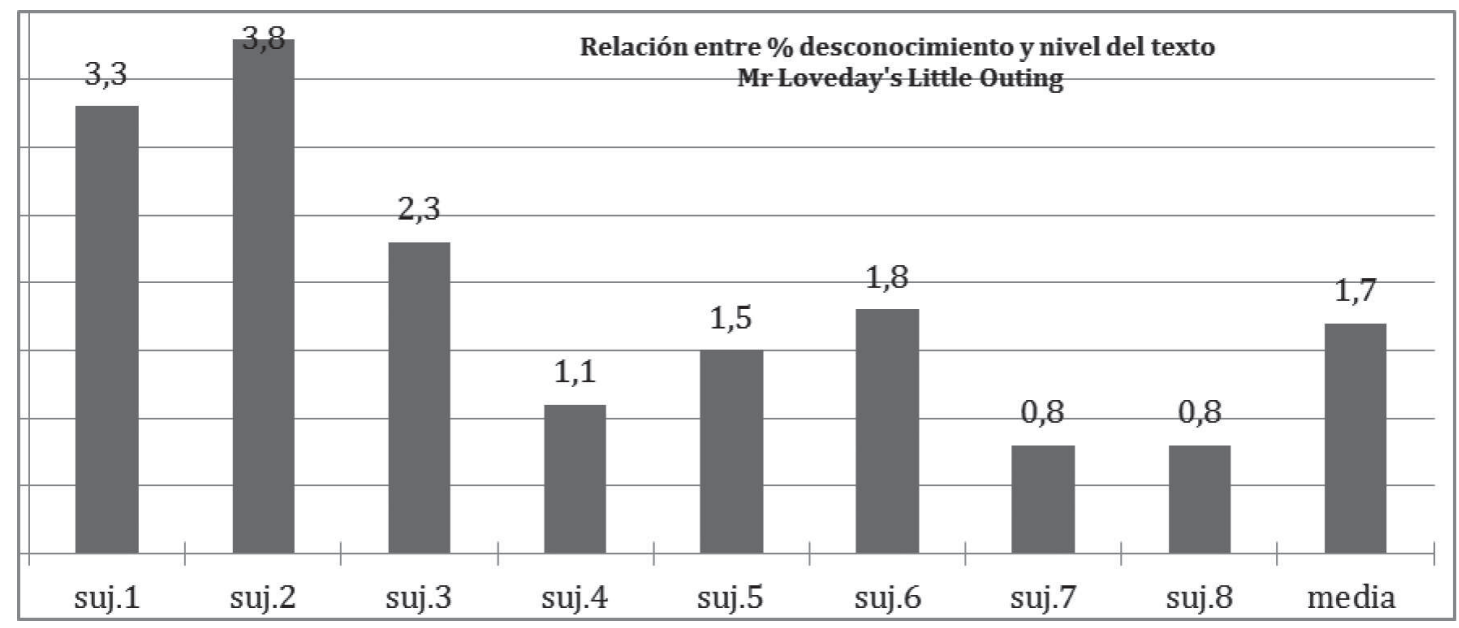

Gráfico 4. Relación desconocimiento y nivel de texto en Mr. Loveday's Little Outing. 


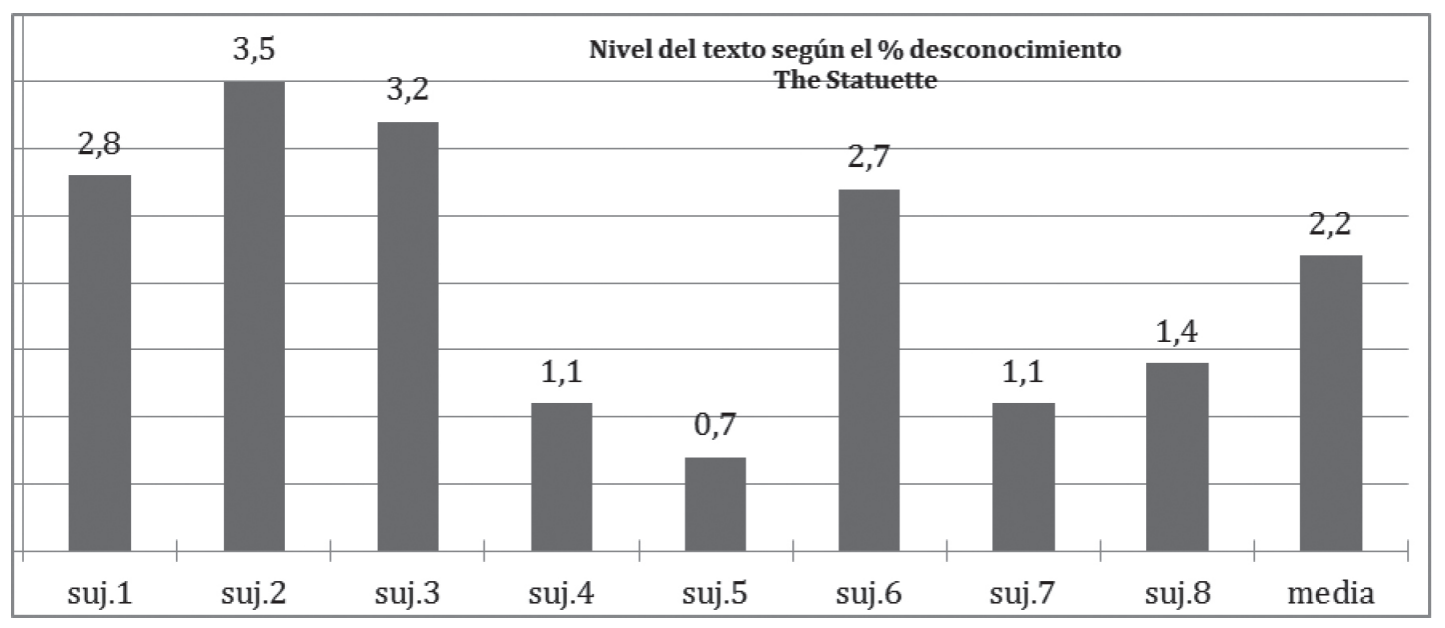

Gráfico 5. Relación desconocimiento y nivel de texto en The Statuette.

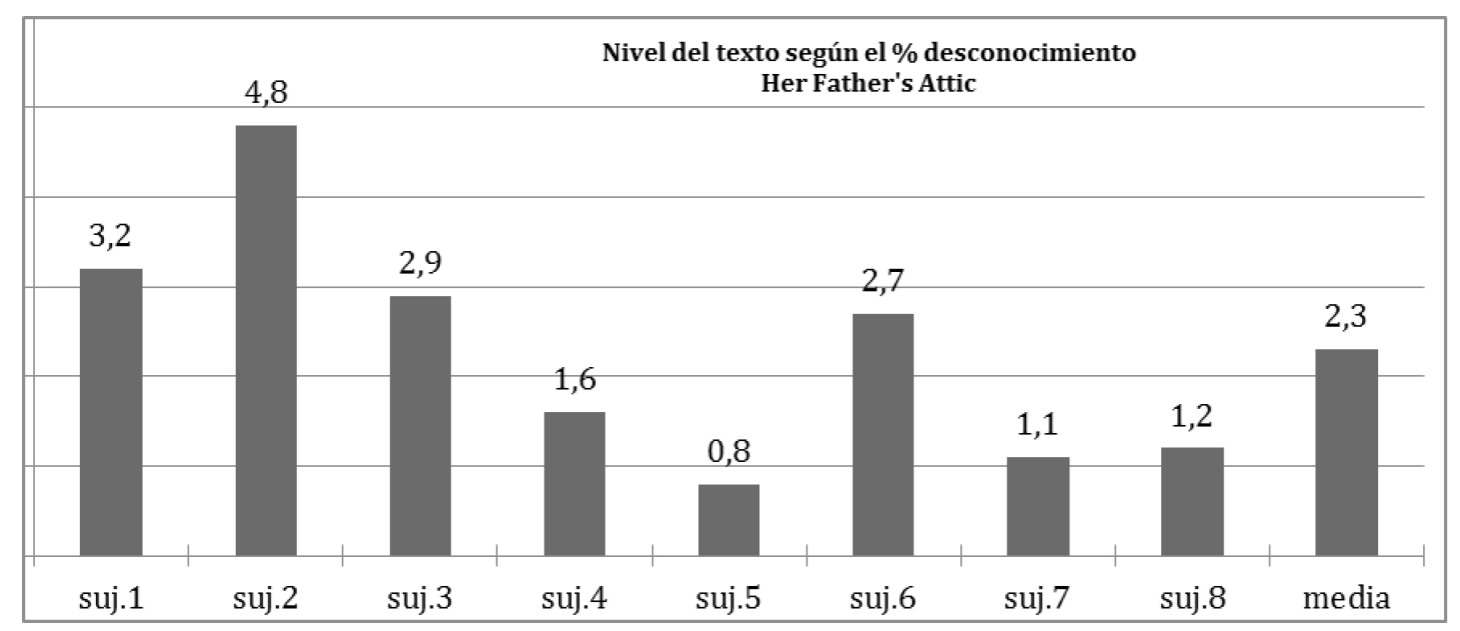

Gráfico 6. Relación desconocimiento y nivel de texto en Her Father's Attic.

Si nos centramos inicialmente en la media de cada una de las historias, salta a la vista cómo la más difícil de las tres ha sido Her Father's Attic (2,3\%), seguida de The Statuette (2,2\%) y de Mr. Loveday's Little Outing (1,7\%). Por lo tanto, dos de las tres historias cortas que conforman el corpus de estudio tienen un nivel difícil. Individualmente, para los sujetos 1, 2 y 3 el nivel de lectura es difícil (destacando el sujeto 2 en las tres historias - 3,8, 3,5 y 4,8\% respectivamente). Para el sujeto 6, la primera historia está un poco por encima de lo que se puede considerar como apropiado $(L+1)$. Sin embargo, la segunda y la tercera lectura se corresponden con un nivel difícil. Para el resto de sujetos (4, 5, 7 y 8) las historias son fáciles, apropiadas o $\mathrm{L}+1$. Estos datos coinciden con las conclusiones aportadas en la cobertura léxica del texto. Mr. Loveday's Little Outing presenta un nivel fácil para los sujetos 7 y 8 , y apropiado para los sujetos 4 y 5 . Tal vez por ello sus diarios 
de lectura correspondientes a estas historias sean más profundos que los del resto. The Statuette sólo le ha resultado fácil al sujeto 5 y apropiada a los sujetos 4, 7 y 8. Finalmente, en Her Father's Attic, el nivel de la lectura es de nuevo fácil para el sujeto 5. Su diario de lectura, especialmente en esa historia, aporta un alto grado de implicación y de significación para el sujeto.

\subsection{Estrategias utilizadas durante la lectura para el acceso al significado}

Por último, se utilizará la información de los diarios de lectura y que arroja luz acerca de las estrategias empleadas para acceder al significado de las palabras desconocidas. El uso de estas estrategias, dentro del esquema del proceso de lectura, se situaría en las fases de descodificación, precomprensión y comprensión.

Los sujetos investigados reconocen mayoritariamente (54\%) hacer uso del contexto léxico de la palabra para acceder a su significado (estrategias del procesamiento descendente). Para que sea posible deducir una palabra por el contexto, es necesario que el resto de palabras que lo configuran (es decir el marco léxicocontextual) sea familiar para el lector. La deducción contextual, por un lado, les permite mantener la fluidez lectora, el ritmo de lectura, la concentración, la naturalidad de la lectura. No obstante, la deducción va a ir cargada de un cierto toque de suposición que se traduce en una imprecisión semántica.

Otras estrategias para la solución de cuestiones léxicas han sido la búsqueda en el diccionario (31\%) o simplemente ignorar el término (15\%). Las razones que conducen a los sujetos lectores a buscar palabras en el diccionario (estrategia del procesamiento ascendente) pueden ser de muy diferente índole. Por un lado, puede que ese término aparezca aleatoriamente en varias partes del texto. Esta repetición suele originar en el lector una necesidad de precisión léxica y, para solucionar tal estado de curiosidad, recurre al diccionario. Otras veces, el vocablo es familiar para el sujeto lector (seguramente de lecturas anteriores), pero no recuerda su significado. En este caso, posiblemente decida refrescar su mente por medio de la búsqueda en el diccionario. En otras ocasiones, el uso del diccionario responde a criterios difícilmente catalogables (combinación ortográfica de la palabra, curiosidad léxica...).

Tal y como se expuso anteriormente, para que el contexto de un término sea efectivo es necesario que las palabras que lo componen sean conocidas. En el caso contrario, donde el mismo contexto presenta dudas semánticas, el sujeto tiene varias opciones. Por un lado, si el término léxico no es relevante, desde su punto de vista, para seguir leyendo, posiblemente lo ignore (estrategia del procesamiento descendente) y continúe la lectura. Si la palabra representa cierta relevancia en ese punto de lectura, probablemente utilice la estrategia de búsqueda en el diccionario. 


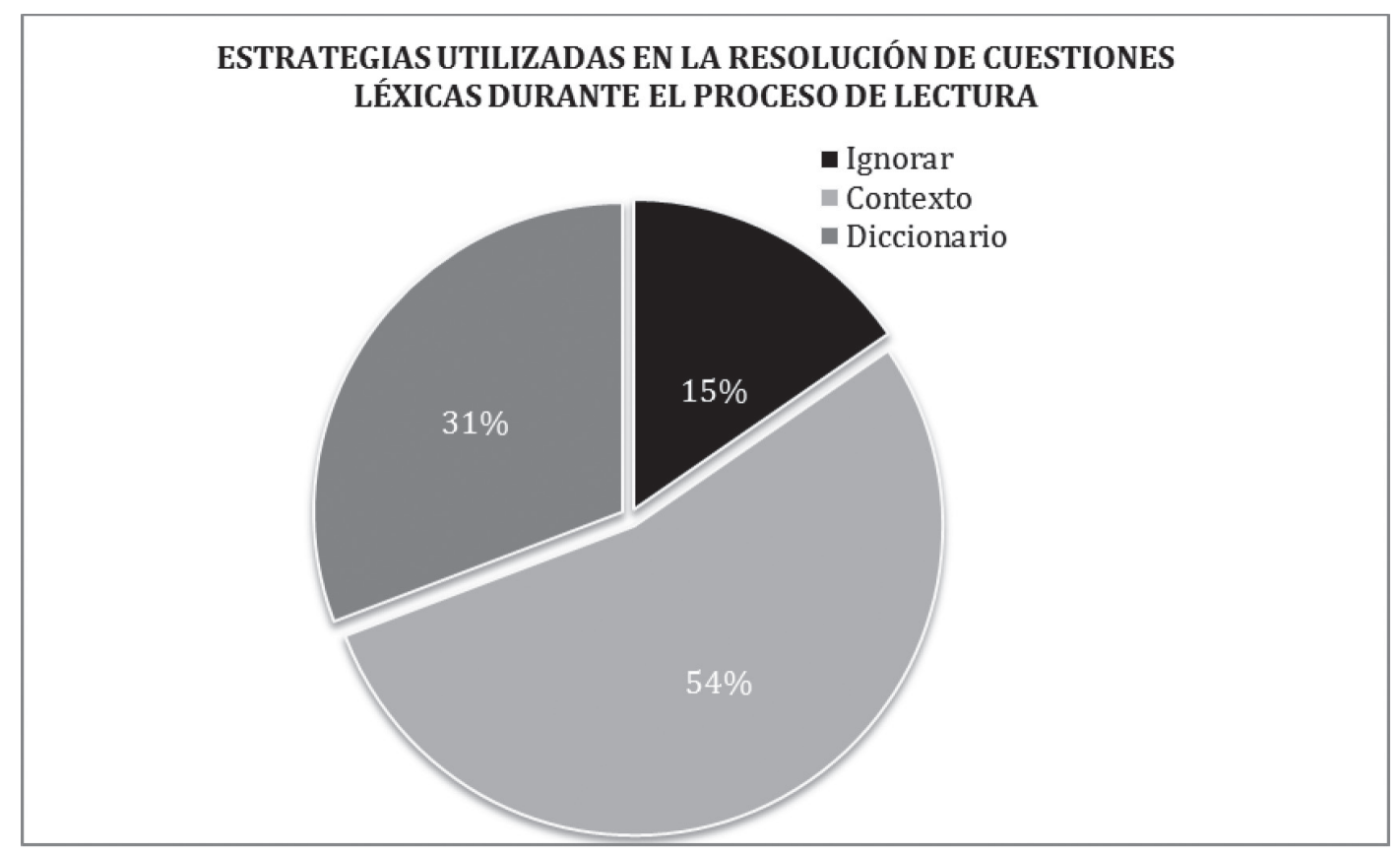

Gráfico 7. Estrategias utilizadas en resolución de cuestiones léxicas.

\section{CONCLUSIONES Y REFLEXIONES FINALES}

El objetivo inicial de este artículo era valorar el léxico en relación con la comprensión e interpretación de textos en una lengua extranjera. Tras llevar a cabo la investigación y analizar los datos cuantitativos que se reflejan en el apartado anterior, podemos apreciar cómo el área semántica se erige, una vez más, como la principal a la hora de establecer los niveles de dificultad. Aunque otros campos de la lingüística son también importantes (sintaxis, pragmática...), el poder connotativo y denotativo de las palabras es incomparablemente superior en la comprensión del texto. Así, la muestra que se ha utilizado para la investigación asegura que es necesario conocer un 96\% (mínimo) del vocabulario textual si se pretende acceder al significado. Los momentos en los que ese porcentaje ha sido igual o superior, la información se ha asimilado con más detalles y se ha recuperado más fácilmente en la entrevista. De igual forma, hay un paralelismo en los participantes que no han llegado en algunos de los textos al mínimo establecido. La información era mucho más vaga y sus diarios de lectura, tal y como se verá en una próxima publicación, se sitúan a un nivel muy superficial (todas estas reflexiones se recogen en los diarios de lectura que los participantes entregaron y donde se perseguía una aproximación individual y cualitativa al proceso lector en la lengua extranjera).

Para el docente de lengua extranjera que pretenda desarrollar el hábito lector en sus estudiantes, es fundamental que los textos se ubiquen dentro del rango consensuado por la comunidad científica tal y como demuestra esta investigación. 
Si, por el contrario, el texto es demasiado difícil, se verán obligados a recurrir frecuentemente al diccionario (lo cual interrumpe y fragmenta la trama textual). Además, la motivación se ve seriamente afectada, especialmente entre el alumnado de primaria y secundaria con unos intereses bastante dispersos en cuestión de segundas lenguas. Los textos "indescifrables" se convierten más en un castigo que en un beneficio a largo plazo y el estudiante desarrolla actitudes poco alentadoras para el desarrollo lingüístico. Los participantes en el estudio manifestaron haber prestado más atención a los textos que habían comprendido mejor al poder relacionarlos más fácilmente con el conocimiento almacenado en el intertexto (experiencias previas cuyos esquemas mentales se activan al entrar en contacto con situaciones análogas). Por lo tanto, sea cual sea el nivel de competencia lingüística del sujeto, el texto (o textos) al que se vea expuesto tiene que estar dentro de su cobertura óptima de vocabulario pasivo.

Las limitaciones del estudio estriban, principalmente, en el reducido número de textos al que los estudiantes se vieron expuestos. Dado que esta investigación constaba de dos partes, una cuantitativa que se expone aquí y otra cualitativa que se publicará en breve, los textos tuvieron que limitarse a tres historias cortas. Futuras investigaciones podrían focalizarse en el desarrollo de un programa de lectura mucho más abundante donde los estudiantes aprecien las repercusiones de la experiencia en su competencia lingüística y nosotros, como investigadores, avancemos en los resultados que se exponen en este trabajo.

\section{REFERENCIAS}

Carter, R. y McCarthy, M. (1988). Vocabulary and language learning. Essex: Longman.

Carver, R.P. (1994). Percentage of unknown vocabulary words in text as a function of the relative difficulty of the text: Implications for instruction. Journal of Reading Behavior, 26 (4), 413-437.

Cobb, T. y Horst, M.E. (2004). Is there room for an Academic word list in French. En P. Bogaards y B. Laufer (eds.), Vocabulary in a second language (pp. 15-38). Amsterdam: John Benjamins.

Council of Europe. (2001). Common European framework for languages: Learning, teaching, assessment. Oxford: Oxford University Press.

Day, R. y Bamford, J. (2000). Extensive reading in the second language classroom. Cambridge: Cambridge University Press.

Freebody, P. y Anderson, R. C. (1983). Effects of vocabulary difficulty, text cohesion and schema availability on reading comprehension. Reading Research Quarterly, 18 (3), 233-94.

Gil Flores, J. (2011). Hábitos lectores y competencias básicas en el alumnado de 
educación secundaria obligatoria. Educación XXI, 14 (1), 117-134.

Golberg, H., Paradis, J. y Crago, M. (2008). Lexical acquisition over time in minority first language children learning English as a second language. Applied Psycholinguistics, 29, 41-65.

Grabe, W. y Stoller, F. L. (2001). Reading for Academic Purposes: Guidelines for the ESL/EFL Teacher. En Marianne Celce-Murcia (ed.) Teaching English as a second foreign language (pp. 187-203). London: Heinle \& Heinle.

$\mathrm{Hu}$, M. \& Nation, P. (2000). Unknown vocabulary density and reading comprehension. Reading in a Foreign Language, 13 (1), 403-430.

Ireland, K. (2001). The Statuette. En D. Pepper (ed.), Nasty endings I (pp. 79-88). Oxford: Oxford University Press.

Krashen, S. D. (1982). Principles and practice in second language acquisition. New York: Prentice Hall.

Krashen, S. D. (1993). The power of reading. Insights from the research. Englewood Colorado: Libraries Unlimited, Inc.

Laufer, B. y Ravenhorst-Kalovski. (2010). Lexical threshold revisited: lexical text coverage, learners' vocabulary size and reading comprehension. Reading in a Foreign Language, 22, 15-30.

Laufer, B. y Sim, D.D. (1985a). An attempt to measure the threshold of competence for reading comprehension. Foreign Language Annals, 18 (5), 405-411.

Laufer, B. y Sim, D.D. (1985b). Taking the easy way out: non-use and misuse of contextual clues in EFL reading comprehension. English Teaching Forum, 23 (2), 7-20.

Nation, P. (1997). The language learning benefits of extensive reading. The Language Teacher Online, 21 (5). [en línea]. Disponible en http://jalt-publications. org/old_tlt/files/97/may/benefits.html. Consulta: 23.02.2013.

Nation, P. (2001). Learning vocabulary in another language. Cambridge: Cambridge University Press.

Nation, P. (2006). How large a vocabulary is needed for reading and listening? The Canadian Modern Language Review/La revue canadienne des langues vivantes, 63, 59-81.

Nation, P. y Coady, J. (1988). Vocabulary and reading. En R. Carter y M. McCarthy (eds.), Vocabulary and language teaching (pp. 97-110). Essex: Longman.

Pearce, P. (2002). Her Father's Attic. En P. Pearce, Familiar and haunting: Collected stories. New York: GreenWillow Books.

Pérez Basanta, C. (2005). Assessing the receptive vocabulary of Spanish students of English philology: An empirical investigation. En J.L. Martínez Dueñas, N. McLaren, C. Pérez Basanta y L. Quereda Rodríguez-Navarro (eds.), Towards an understanding of the English language: past, present and future. Studies in honour of Fernando Serrano (pp. 1-21). Granada: Editorial Universidad de Granada. 
Ruiz-Cecilia, R. (2006). El diario de lectura como instrumento didáctico para el aprendizaje de lenguas extranjeras. Lenguaje y Textos, 23-24, 187-196.

Samuels, S. J. (1994). Toward a theory of automatic information processing in reading, revisited. En R. B. Ruddell, M. R. Ruddell y H. Singer (eds.), Theoretical models and processes of reading (pp. 816-837). Newark: International Reading Association.

Schmidt, R. (1990). The role of consciousness in second language learning. Applied Linguistics, 11 (2), 29-158.

Terrazas Gallego, M. y Agustín Llach, M.P. (2009). Exploring the increase of receptive vocabulary knowledge in the foreign language: a longitudinal study. International Journal of English Studies, 9 (1), 113-133.

Ulijn, J. M. y Kempen, G. A. M. (1976). The role of the first language in second language reading comprehension: Some experimental evidence. En G. Nickel (ed.), Proceedings of the $4^{\text {th }}$ International Congress of Applied Linguistics (pp. 495-507). Stuttgart, Germany: Hochschulverlag.

Waring, R., y Takaki, M. (2003). At what rate do learners learn and retain new vocabulary from reading a graded reader? Reading in a Foreign Language, 15 (2), 130-163.

Waugh, E. (2003). Mr Loveday's Little Outing. En C. West (ed.), From the cradle to the grave (pp. 19-30). Oxford: OUP.

Webb, S. (2008). Receptive and productive vocabulary sizes of L2 learners. Studies in Second Language Acquisition, 30, 79-95. 
Anexo I

\begin{tabular}{|c|c|c|c|c|c|c|c|c|c|}
\hline \multirow[b]{2}{*}{ SUJETOS } & \multicolumn{3}{|c|}{$\begin{array}{l}\text { FRECUENCIAS ABSOLUTAS } \\
\text { Mr Loveday's Little Outing }\end{array}$} & \multicolumn{3}{|c|}{$\begin{array}{l}\text { FRECUENCIAS ABSOLUTAS } \\
\text { The Statuette } \\
\end{array}$} & \multicolumn{3}{|c|}{$\begin{array}{c}\text { FRECUENCIAS ABSOLUTAS } \\
\text { Her Father's Attic }\end{array}$} \\
\hline & $\begin{array}{c}\text { No DE } \\
\text { PALABRAS } \\
\text { DESCONOCIDAS }\end{array}$ & $\begin{array}{c}\text { No DE } \\
\text { PALABRAS } \\
\text { CONOCIDAS }\end{array}$ & $\begin{array}{l}\text { No TOTAL } \\
\text { DE } \\
\text { PALABRAS }\end{array}$ & $\begin{array}{c}\text { No DE } \\
\text { PALABRAS } \\
\text { DESCONOCIDAS }\end{array}$ & $\begin{array}{c}\text { No DE } \\
\text { PALABRAS } \\
\text { CONOCIDAS }\end{array}$ & $\begin{array}{c}\text { No TOTAL } \\
\text { DE } \\
\text { PALABRAS }\end{array}$ & $\begin{array}{c}\text { No DE } \\
\text { PALABRAS } \\
\text { DESCONOCIDAS }\end{array}$ & $\begin{array}{c}\text { No DE } \\
\text { PALABRAS } \\
\text { CONOCIDAS }\end{array}$ & $\begin{array}{l}\text { No TOTAL } \\
\text { DE } \\
\text { PALABRAS }\end{array}$ \\
\hline Suj. 1 & 87 & 2560 & 2647 & 80 & 2825 & 2905 & 70 & 2113 & 2183 \\
\hline Suj. 2 & 100 & 2547 & 2647 & 130 & 2775 & 2905 & 105 & 2078 & 2183 \\
\hline Suj. 3 & 60 & 2587 & 2647 & 93 & 2812 & 2905 & 64 & 2119 & 2183 \\
\hline Suj. 4 & 30 & 2617 & 2647 & 31 & 2874 & 2905 & 35 & 2148 & 2183 \\
\hline Suj. 5 & 40 & 2607 & 2647 & 20 & 2885 & 2905 & 18 & 2165 & 2183 \\
\hline Suj. 6 & 48 & 2599 & 2647 & 77 & 2828 & 2905 & 58 & 2125 & 2183 \\
\hline Suj. 7 & 20 & 2599 & 2647 & 31 & 2874 & 2905 & 25 & 2158 & 2183 \\
\hline Suj. 8 & 20 & 2627 & 2647 & 39 & 2866 & 2905 & 27 & 2156 & 2183 \\
\hline
\end{tabular}

\begin{tabular}{|c|c|c|c|c|c|c|c|c|c|c|c|c|}
\hline \multirow{3}{*}{ SUJETO } & \multicolumn{4}{|c|}{ Mr Loveday's Little Outing } & \multicolumn{4}{|c|}{ The Statuette } & \multicolumn{4}{|c|}{ Her Father's Attic } \\
\hline & \multirow{2}{*}{$\begin{array}{c}\text { TASA DE } \\
\text { PALABRAS } \\
\text { DESCO- } \\
\text { NOCIDAS }\end{array}$} & \multicolumn{2}{|c|}{ FRECUENCIAS RELATIVAS } & \multirow[b]{2}{*}{ NIVEL } & \multirow{2}{*}{$\begin{array}{c}\text { TASA DE } \\
\text { PALABRAS } \\
\text { DESCO- } \\
\text { NOCIDAS }\end{array}$} & \multicolumn{2}{|c|}{ FRECUENCIAS RELATIVAS } & \multirow[b]{2}{*}{ NIVEL } & \multirow{2}{*}{$\begin{array}{c}\text { TASAS DE } \\
\text { PALABRAS } \\
\text { DESCO- } \\
\text { NOCIDAS }\end{array}$} & \multicolumn{2}{|c|}{ FRECUENCIAS RELATIVAS } & \multirow[b]{2}{*}{ NIVEL } \\
\hline & & $\begin{array}{l}\text { \% DE DES- } \\
\text { CONOCI- } \\
\text { MIENTO }\end{array}$ & $\begin{array}{l}\text { COBERTURA } \\
\text { (COVERA- } \\
\text { GE) } \%\end{array}$ & & & $\begin{array}{l}\% \text { DE DES- } \\
\text { CONOCI- } \\
\text { MIENTO }\end{array}$ & $\begin{array}{l}\text { COBERTURA } \\
\text { (COVERA- } \\
\text { GE) } \%\end{array}$ & & & $\begin{array}{l}\text { \% DE DES- } \\
\text { CONOCI- } \\
\text { MIENTO }\end{array}$ & $\begin{array}{c}\text { COBERTURA } \\
\text { (COVERAGE) \% }\end{array}$ & \\
\hline Suj. 1 & $1 / 30$ & 3,3 & 96.7 & Difícil & $1 / 36$ & 2,8 & 97,2 & Difícil & $1 / 32$ & 3,2 & 96,8 & Difícil \\
\hline Suj. 2 & $1 / 26$ & 3,8 & 96,2 & Difícil & $1 / 21$ & 4,5 & 95,5 & Difícil & $1 / 21$ & 4,8 & 95,2 & Difícil \\
\hline Suj. 3 & $1 / 44$ & 2,3 & 97,7 & Difícil & $1 / 31$ & 3,2 & 96,8 & Difícil & $1 / 34$ & 2,9 & 97,1 & Difícil \\
\hline Suj. 4 & $1 / 88$ & 1,1 & 98,9 & Apropiado & $1 / 94$ & 1,1 & 98,9 & Apropiado & $1 / 63$ & 1,6 & 98,4 & $\mathrm{~L}+1$ \\
\hline Suj. 5 & $1 / 66$ & 1,5 & 98,5 & Apropiado & $1 / 145$ & 0,7 & 99,3 & Fácil & $1 / 121$ & 0,8 & 99,2 & Fácil \\
\hline Suj. 6 & $1 / 55$ & 1,8 & 98,2 & $\mathrm{~L}+1$ & $1 / 38$ & 2,7 & 97,3 & Difícil & $1 / 38$ & 2,7 & 97,3 & \begin{tabular}{|l|} 
Difícil \\
\end{tabular} \\
\hline Suj. 7 & $1 / 132$ & 0,8 & 99,2 & Fácil & $1 / 94$ & 1,1 & 98,9 & Apropiado & $1 / 87$ & 1,1 & 98,9 & Apropiado \\
\hline Suj. 8 & $1 / 132$ & 0,8 & 99,2 & Fácil & $1 / 75$ & 1,4 & 98,6 & Apropiado & $1 / 80$ & 1,2 & 98,8 & Apropiado \\
\hline Media & $1 / 59$ & 1,7 & 98,3 & $\mathrm{~L}+1$ & $1 / 46$ & 2,2 & 97,8 & Difícil & $1 / 44$ & 2,3 & 97,7 & Difícil \\
\hline
\end{tabular}

